\title{
Index to Volume 15
}

Absorption 822

Abu-Osba, Y. K. 143

Acidemia 870

Adam, P. A. J. 859, 945

Adams, A. J. 1345

Adams, H. 1097

Adcock, E. W., III 357

Adenine nucleotide 1478

Adenylate cyclase 1083, 1105

ADH 278

Adipocyte 1381

Adolescent 1024

Age 123, 1032, 1340

Age-dependent enzymes 133

Ahlström, H 979

Ailabouni, A. H. 250

Airozo, D. 1478

Aladjem, M. 282

Albumin 1425

Aldosterone 163, 1419

Allometry 1370,1375

Alpers, D. H. 1473

Alpha-naphtyl acetate esterase 1266

Alpha-thalassemia 1093

Altay, C. 1093

Alpha-amylase 940

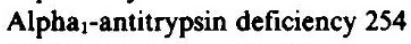

Alpha ${ }_{2}$-macroglobulin 839

Alpha fetoprotein 50

Alter, B. P. 1036

Alveolar macrophages 229

Alveolar morphometry 235

Alveoli 874

Amino acid 115, 357

Aminoglycoside 1234

Aminoisobutyric acid 152

Aminonucleoside 340

Amlie, R. N. 295

Ammonia 1473

Amniotic fluid 996, 1419

Andria, G. 70

Anemia of prematurity 1500

Angiotensin 1088

Angiotensinase 1088

Animal models 128

Ank, B. J. 1256

Anthropometry 1390

Antibody, monoclonal 1282

Antigen 241, 245

Antithrombin III 1015

Apnea 367,870

Arant, B. S., Jr. 1513

Arterial occlusion 143

Arteries, pulmonary 1397

Artificial feeding 245

Asphyxia 44, 53, 1415, 1429

Aspirin 1293

Assael, B. M. 304

Assailly, C. 326

Asthma 1433, 1520

ATP 34

ATPase, myosin 1128

Auricchio, S. 885, 991
Autonomic nervous system, 1055

Autooxidation 794

Baehner, R. L. 1356

Bailey, P. E. 1024

Bailie, M. D. 1088

Baker, L. 1504

Ballabriga, A. 798

Ballejo, G. 850

Balsam, A. 1123

Baluarte, H. J. 853

Banchini, G. 1073

Barber, A. 1454

Bard, H. 967, 1500

Barnicle, N. A. 335

Barton, T. P. 905

Bartter's syndrome 926

Baskan, N. 299

Bastomsky, C. H. 169

Bateman, D. 1111

Battaglia, F. C. 1340

Baumgart, S. 1495

Beatty, C. H. 1105

Beck, J. C. 235

Becker, K. L. 1032

Bégin, R. 809

Behavioral activity 1509

Benson, P. F. 959

Ben-Yoseph, Y. 839

Beratis, N. G. 257

Berryman, C. C. 62

Berthiaume, Y. 809

Bertin, R. 787

Bertolino, F. 1443

Bertorini, T. E. 1478

Beta-adrenergic receptors, 1083, 1363

Beta-amino acid 10

Betamethasone 241

Betke, K. 53

Bhat, R. 1406

Bhutani, V. K. 829

Bicknell, D. 1282

Bile salt 1425

Bilirubin 345, 1009, 1517

Blake, D. A. 1293

Blood 971, 1045

Blood flow 874

Blood flow, cerebral 1415

Blood resistivity 964

Blood volume 53

Bloor, C. M. 147

Blum, P. M. 1256

Boat, T. F. 1234

Bocek, R. M. 1105

Body composition 847

Bohr effect 809

Bonati, M. 304

Bone marrow 47

Bone marrow culture 95

Boney, F. 1439

Book, L. 50

Borden, M. 1447

Bortolussi, R. 1287
Borulf, S. 1051

Bouchlas, D. 817

Bourdony, C. J. 6

Boutellier, U. 28

Boxer, L. A. 229

Boxer, L. A. 1356

Bracke, P. 58

Brain 319, 335, 859, 1415, 1429

Brain cell 1282

Brain edema 44

Brandtzaeg, P. 1134

Brans, Y. W. 1024

Breast milk 1454

Breath hydrogen 1229

Brenner, R. A. 349

Brigham, K. L. 899

Brocklebank, T. 916

Brodrick, J. W. 1351

Bromide, pancuronium, 1097

Bromocriptine 850

Bronchoconstriction 1433

Brooks, D. E. 1429

Brouillette, R. T. 143

Brown, D. R. 1100

Bruck, E. 99

Brush border membrane 112, 214, 99 I

Bucher, J. R., 999

Bureau, M. A. 809

Bustos, R. 850, 996

Butler, J. DeB. 1063

Button, L. R. 959

Calcagno, P. L. 887

Calcitonin 1359

Calcium 803, 985, 1100, 1134 urine 1032

Caldeyro-Barcia, R. 850

Caldicott, W. J. H. 935

Calorimetry 1077

Camden, J. 1439

Campos, J. M. 952

Candida albicans 1287

Cao, A. 1443

Carbohydrate, absorption 1229

Carbohydrates 843, 847, 1068

Carbon dioxide response 1509

Cardiovascular function 935

Cartilage 330

Cassady, G. 1024

Catecholamines 787, 1055, 1483

Cates, D. B. 1509

Cathelineau, L. 974

Cathodal elastase 1051

Cavelier, B. 326

Celiac disease 309, 105I

Cell

pseudoglandular 1303

Chandra, M. 340

Charalampous, S. C. 1019

Chase, P. A. 14

Cheek, D. B. 1370,1375

Cheng, J. B. 1083

Cheng, P. W. 1234 
Chernick, V. 1509

Chesney, R. W. 10

Chessex, P. 1077

Cheung, A. T. W. 1271

Choi, T-S. 1240

Chloramphenical 817

Cholesterol 66, 313

Chondrocyte 330

Chondrocitin sulfates 330

Chromatid exchanges 1019

Chromium-51 905

Chronic renal insufficiency 887

Chrousos, G. P. 1345

Ciliary agglutination 219

Ciliary dyskinesia 219

Circulation, persistent fetal 1397

Clarke, W. R. 1045

Cloutier, M. M. 817

Coagulation 38, 1015

Cole, B. R. 916

Cole, C. H. 926

Coleman, N. 123

Collinge, J. 1040

Compensatory renal growth 1370, 1375

Complement 362, 813

Compton, S. K. 866

Concanavalin A 1314

Concanavalin A capping 1271

Congenital heart disease 1397

Congenital malformations 313

Connective tissue 235

Consamus, B. 1248

Continuous negative pressure 874

Cooper, D. M. 813

Copper 257, 284

Coppes, V. 1429

Coproantibodies 1256

Corbeel, L. 58

Corbo, L. M. 1058

Cordesius, E. 254

Corley, K. P. 157

Cornblath, M. 1345

Cornett, L. E. 1083

Corticosteroids 921,1530

Corticotropin 1261

Cortisol 996

Cortisone 1530

Coudi, F. X. 974

Cowett, R. M. 1348

Cow's milk protein intolerance 1051

Crandell, S. S. 357

Crossley, J. R. 62

Creatine kinase isoenzyme 1128

Creswell, J. S. 1348

Cropp, G. J. A. 1520

Crowley, D. 1397

Cyclic AMP 278, 1009, 1328, 1359, 1363

Cyclic GMP 1359

Cyclic nucleotides 787

Cysteamine, 1063

Cystic fibrosis 14, 120, 839, 940, 1073, 1234, $1308,1328,1351,1439$

Cystic fibrosis factor 940,1073

Cystine 1492

Cystinosis 1063

Cystinosis, nephropathic 1492

Dameron, F. 787

Dancis, J. 1323

Daniel, S. S. 1261

Darovec-Beckerman, C. 1363
David, L. 803

Dean, M. J. 959

De Barsy, T. 58

DeFranco, C. L. 839

de Groot, C. J. 284

Delaval, E. 138

Delivoria-Papadopoulos, M. 34, 133

Dell, R. 1111

Denny, F. W. 1319

Deoxyguanosine 956

Development 10, 269, 349, 787, 853, 866, 916 , 921

Developmental gastroenterology 241, 245

Development 991, 999

postnatal 930

De Virgiliis, S. 1443

De Vizia, B. 991

Devlieger, H. 58

De Vos, C. J. 1303

Diabetes mellitus 62, 1058

Diakomanolis, E. S. 1483

Diarrhea 1009

Dick, M., II 1397

Dickey, D. W. 1520

Diet, 1068

DiGeorge, A. M. 6

Digoxin 282

Dihydroalprenolol 1083

DiMauro, S. 1334

Di Natale 976

Dipalmitoyl phosphatidylcholine 1029

Dipeptidase 357

Dipeptide 357

Dirksen, J. W. 905

Disaccharidase 1009

Disaccharides 930

DNA 916

DNA breaks 1019

Doig, G. 44

Donzelli, G. P. 1517

Dowshen, S. A. 6

Drug

anti-tumor 1117

sensitivity 1117

Drug-induced capping 1314

Duc, G. V. 28, 1140

Duchenne muscular dystrophy 1411,1478

Ducros, R. 921

Ductus arteriosus 19

Dudeja, P. K. 112

Duffy, B. 1040

Duodenum 1051

Duquenne, D. 1433

Durand, M. 1509

Durie, P. R. 1351

Dweck, H. S. 1024

E rosette-forming cells 1266

Eadington, D. W. 1425

ECG 28

E. coli 952

Edgar, B. W. 62

Edwards, N. L. 1478

Eekels, R. 58

Eqami, K. 1370, 1375

Egner, P. G. 1293

Electrolytes 1024

Elliott, R. B. 62

Elphick, M. C. 313

Elzouki, A. Y. 853

Encephalopathy, hypoxic-ischemic 1415
Endotoxin 47, 899

Engle, W. D. 1495

Ennever, J. F. 956

Enterocolitis, necrotizing 1229

Enterocyte 1530

Enzyme activities 112

Enzyme replacement therapy 959

Enzymes, lung 999

Enzymuria 1234

Epidermal growth factor 290

Epinephrine 99, 912, 1381

Erthrocyte 133, 926, 967, 1345, 1425, 1443

Erythrocyte, aplasia 1036

Erythropoiesis 34

Erythropoiesis, fetal-like 1036

Esophageal pressure 1468

Estrogen 1274

Estrous cycle 1488

Ethanol 335

Evans, G. W. 168

Evans, M. A. 1406

Evoked potentials 295

Exocrine pancreatic insufficiency 1073

Extracellular fluid 916

Extracellular water 1024

Fahr, K. 309

Fasting 945

Fat 847

Fat, dietary 1068

Fat/lean tissue 169

Fatty acids, free 1340

Fatty acids, short chain 1097

Faulkner, G. 1287

Feingold, J. 326

Fenoterol 1433

Fetal alcohol syndrome 908

Fetal blood sampling 254

Fetal growth 152

Fetal-like erythropoiesis 1036

Fetal weight 798

Fetus 19, 44, 138, 163, 335, 787, 829, 850, 859, $870,921,967,991,1248,1429$

Fibrinogen 1045

Fibroblast 257, 290, 959, 976, 1063, 1328, $1334,1411,1492$

Field, R. J. 107

Finer, N. 1415

Firek, A. F. 1359

Fischer, G. W. 1525

Fisher, D. A. 1483

Fisher, D. J. 843

Fisher, S. E. 335

Fixler, D. E. 874

Flower, R. W. 1293

Foidart, J-M. 235

Follicle-stimulating hormone 157

Fomon, S. J. 847

Formula milk 822

Forristal, J. 362

Forstner, G. G. 1351

Fouron, J. C. 967,1500

Fowler, R. 1370, 1375

Fox, 1. H. 1478

Fox, W. W. 1495

Fracassa, M. 95

Franzen, B. 254

Fraser, D. 299

Frau, F. 1443

Free fatty acids 1483

Freeman, M. 1429 
Freyse, J. 833

Friedman, A. L. 10

Friedman, W. F. 147, 1128

Free fatty acids 313

Fritze, L. 241, 245

Furzan, J. A. 874

Gabriele, G. 874

Gall, D. G. 1530

Gardey, C. 787

Garry, P. J. 822

Garzon, B. 921

Gaskin, K. J. 1351

Gastric acid 921

Gastrin 803

Gd $^{\text {Mediterranean }}$ phenotype 1443

Geloso, J-P. 138, 921

Genetics 214, 299

Gentamicin 952

Gentile, P. S. 123

Geokas, M. C. 1351

Gestational age 798

Geubelle, F. 1433

Ghishan, F. K. 985

Gilbert, B. A. 822

Ginsberg-Fellner, F. 1381

Girard, J. 1340

Giussi, G. 996

Giussi, G. A. 850

Glomerular density 1248

Glomerular filtration rate 887, 1248

Glössl, J. 976

Glucagon 99, 912

Glucocorticoid hormones 912

Gluconeogenesis 138

Glucocorticoid 99

Glucocorticoids 19

D-Glucose 112

Glucose 250, 313, 319, 945, 1340, 1348

Glucose oligomers 250

Glucose-6-phosphatase 138

Glucose-6-phosphatase deficiency 1504

Glucose-6-phosphate dehydrogenase 1443

Glucose-6-phosphate 133

Glueck, C. J. 66

Glutamine 859,1473

Glutathione peroxidase 1356

Glycine 112, 309, 1454

Glycocholate 1425

Glycogen 1274

Glycogen storage disease type 11345

Glycogenesis 299

Glycogen storage disease 58

Glycoproteins 839

Gmünder, F. K. 1533

Goeringer, G. C. 866

Golden, M. H. N. 1454

Goldfien, A. 1083

Golgi $\alpha$-D-mannosidase 70

Gonadal dysgenesis 157

Gonadotropin-releasing hormone 157

Gout 1447

Grafmeyer, D. C. 803

Granulocyte 1282, 1533

Granulomatous disease, chronic 1533

Gravely, M. E. 1093

Graystone, J. E. 1375

Greaves, M. F. 1282

Green, R. S. 892, 899

Grimer, G. 974

Griscelli, C. 1266
Grodin, W. K. 1055

Groggins, R. C. 22

Grossman, G. 833

Group B $\beta$-streptococcus 892

Group B $\beta$-streptococcus, type III 899

Growth 169, 1390

Growth hormone 99, 1375

Grunting, subclinical 979

Gruskin, A. B. 853

Guanine nucleotides 1363

Guanylate cyclase 1105

Guanylate nucleotides 1105

Guerin, D. M. 1068

Guiraldes, E. 930, 1530

Gunn, T. 1040

Güntner, M. 53

Gupta, C. 1488

Gustavii, B. 254

Guthrie, R. D. 367

Haase, G. 250

Häberle, D. A. 278

Haddad, G. G. 879, 1055

Hair zinc 123

Halkin, H. 282

Hamilton, J. R. 930, 1530

Harker, L. C. 147

Harnaes, K. 1134

Harries, J. T. 1073

Harris, H. B. 1024

Harris, M. C. 952

Harrison, G. M. 940

Harrison, M. R. 1229

Hart, M. J. 1314

Haschke, F. 847

Hathaway, W. E. 1015

Hay, W. W., Jr. 1015

Heart development 1128

Heart disease, congenital 1397

Heart rate 1077, 1111

Hees, P. 1397

Hegarty, P. V. J. 128

Heidelberger, K. 1397

Height 123

Heim, T. 1077

Heldt, G. P. 1229

Helinek, G. L. 985

Hellerqvist, C. G. 892,899

Hematocrit 964

Hematolog disorders 1093

Hemodynamics 899

Hemoglobin 822

Henning, S. J. 1068

Heparin 1015

Hepatocyte 1406

Herin, P. 1248

Herrington, P. T. 1105

Herschkowitz, N. 319

Herschkowitz, N. N. 1411

Heymann, M. A. 843

Higgins, C. 1356

High-density lipoprotein 66

Hill, B. T. 1117

Hirschhorn, K. 257

Histocompatibility 62

HLA system 326, 959

Hobel, C. J. 1483

Hodges, G. C. 22

Hofstad, F. 1134

Holt, A. B. 1370

Holzman, I. 335
Hooper, E. M. 822

Hormone parathyroid 1134

Howland Award 1323, 1327

Hoyer, J. R. 340

Hue, L. 58

Human growth hormone 6

Human immune globulin 1256

Human milk 822

Hume, R. 1425

Hunter, K. W. 1525

Hurley, L. S. 166

Husain, M. K. 1261

Hutchins, G. M. 235

Hutson, J. M. 1370, 1375

Hyperalphalipoproteinemia 66

Hyperammonemia 1473

Hyperbilirobinemia 1019, 1443, 1517

Hypercalcitoninemia 803

Hypercapnea 1402

Hyperinsulinemia 1422

Hyperlipidemia 887

Hyperoxia 262, 999, 1356, 1402

Hypertension 935

Hypertriglyceridemia 1504

Hyperuricemia 1447

Hyperviscosity 1348

Hypocalcemia 1100

Hypocomplementemia 362

Hypoglycemia 859, 912, 945, 1345, 1422, 1504

Hypomagnesemia 1134

Hypometabolism 169

Hypopituitarism 912

Hypophysial hormones 138

Hypopituitarism 6

Hypothyroidism 169

Hypoxanthine 28, 1140

Hypoxemia 367,870

Hypoxia 28, 367, 1140, 1520

Hypoxia, intrauterine 1397

Hypoxic-ischemic encephalopathy 1415

Iancu, T. C. 299

I-cell disease 70, 330, 1334

IgG 1256

IgM 219

Ileum 1473

Immune complexes 362

Immunity 908

Immunofixation 254

Immunoglobulins A, G, and M 813

Impedance cardiography 964

Impero, J. E. 940

Indomethacin 147, 278, 1406

Infant 50, 1024

Infertility 1488

Ingbar, S. H. 1123

Ingelfinger, J. R. 935

Ingwall, J. S. 1128

Inotropic state 349

Inoue, S. 95

Inspiration:expiration 833

Insulin 798, 1348, 1381, 1513

Insulin receptors $1058,1303,1345$

Intestinal immunity 1256

Intestine 112, 241, 245, 250, 309, 345, 985, $1068,1073,1530$

Intestine, small 930, 991, 1009

Intracellular fluid 916

Intracellular water 1024

Intracranial pressure 1097

Intra-uterine growth retardation 53 
Ion-transport 1530

Iron $\mathbf{8 2 2}$

Ischemia, intestinal 1229

Isoproterenol 1328

Islet cell antibodies

Jackson, A. A. 1454

Jacobson, L. J. 1015

Jaeken, J. 58

Jakubowski, A. F. 912

James, A. G. 62

James, L. S. 1111

James, L. S. 1261

Jap, G. H. 53

Jaundice 345

Jaundice phototherapy 1009

Jax, D. K. 10

Jejunum 1530

Jeppsson, J-O. 254

Johnson, B. 979

Johnson, F. 22

Johnson, G. S. 1356

Johnson, J. H. 1351

Johnson, J. W. C. 235

Johnson, S. 908

Johnston, R. B., Jr. 1465

Jonas, A. J. 1492

Joppick, R. 278

Jose, P. A. 887

Joseph, B. R. 1093

Juvenile rheumatoid arthritis 1266

Kairam, R. 1111

Kallidin 1439

Kamoun, P. P. 974

Kanarek, D. J. 1402

Kaplan, S. A. 1058

Kaplinsky, C. 282

Karl, I. E. 1473

Katz, S. 257

Kaufman, I. A. 1447

Kelck, R. P. 157

Kelly, D. H. 1402

Kemshead, J. T. 1282

Kerzner, B. 250

Ketoacidosis 99

Ketone 1340

Khosla, S. S. 1274

Kida, K. 269

Kidney $10,138,163,278,282,340,916,1088$, $1100,1234,1303,1359,1370,1375$, 1513

Kidney mass 935

Kidney transplant 935

Kim, D. H. 1359

Kim, K. O. 128

Kimura, G. M. 1271

Kinase 229

King, M. 120

Kirklin, J. W. 1024

Kirkpatrick, S. E. 147

Kisker, C. T. 1045

Kleinman, R. 241

Kliegman, R. M. 859, 945

Knight, R. 908

Knittle, J. L. 1381

Knowles, P. J. 1429

Koller, E. A. 28

Kraemer, R. 1433

Krall, J. M. 1390

Kramer, M. F. 1128
Krantman, H. J. 1256

Krasilnikoff, P. A. 214

Krebs, R. E., Jr. 47

Kresse, H. 976

Krieger, I. 95

Krugman, S. 1327

Kumar, S. P. 34, 133

Kurz, J. B. 1328

La Badie, G. U. 257

Lachmann, B. 833

Lai, T. L. 879

Lactase 345

Lactate $28,319,1140,1340$

Lactate dehydrogenase 1128

Lactic acidosis 1504

Lamellar bodies 1274

Lando, H. M. 1032

Largman, C. 1351

Laser photolysis 1517

Latini, R. 304

Laufer, Y. 282

Leahy, F. N. 1509

Leake, R. D. 1271

Lebenthal, E. 1240

Lecithin 850, 996

Lederer, B. 58

Ledosseur, P. 326

Lee, P. C. 1240

Lee, S. H. S. 1287

Lefort, J. 326

Le Guennec, J. C. 967,1500

Leistner, H. L. 879, 1055

Lerner, A. 299

Leucine 335

Leucine sensitivity 1345

Leukocyte 38, 1063

Lew, C. D. 1422

Lewy, J. E. 340

Liechti-Gallati, S. 1411

Light, visible 956, 1019

Lindberg, T. 1051

Linderkamp, 0.53

Lindroth, M. 979

Linear growth 123

Link, M. P. 1036

Lipoproteins 887

Liver 299, 335, 1088, 1123, 1303, 1504

Löfberg, L. 254

London, W. T. 235

Lönnerdal, B. 163

Low birth weight infant 803

Low-compliance lung disease 1468

Lowe, C. U. 203, 205

Lung $147,262,269,787,829,833,850,860$, $892,899,979,999,1029,1058,1245$, $1274,1303,1356,1402,1433,1520$

Lung, disease, low-compliance 1468

Lung volume 235

Luteinizing hormone 157

Lutz, P. 309

Lymphocyte 38, 813, 1287

Lymphocyte culture 14

Lysinuric protein intolerance 115

Lysosomal hydrolases 70

Lysosome 1334

MacCallum, M. 1509

MacGillivray, M. H. 99, 912

Macrophage 1356

Mahmood, A. 112
Maisels, M. J. 1468

Mangos, J. A. 817

Mannosidosis 70

Marder, H. K. 362

Marin, L. 787

Marmer, D. J. 908

Maroteaux, P. 976

Marra, G. 304

Marshall, J. C. 157

Martinez, J. R. 1439

Martinez, R. 1308

Mason, D. T. 349

Mass, I. R. 870

Maxwell Frick equation 964

Mayock, D. E. 367

McCarty, G. E. 1429

McClung, H. J. 250

McCurdy, R. E. 1308

McDonald, M. M. 1015

McLeod, D. S. 1293

Meban, C. 1029

Mechanically-ventilated neonates 1468

Medullary thyroid carcinoma 1032

Magnesium 803, 985, 1100, 1134

Mellins, R. B. 879, 1055

Membrane, brush border 991

Meningitis 908

Meningomyelocele 326

Menkes' disease 257, 284

Metabolic acidosis 809

Metabolic rate 1077

Metabolism 6, 34, 133, 304, 319, 335, 803, 839, $843,859,945,1040,1406$

Metabolism, energy 1128

Metallothionein 257, 284

Methylglucose 152

Methylmalonic acidemia 95

Miettinen, E. L. 859, 945

Migliorini, M. G. 1517

Milk formula 1240

Milla, P. J. 1073

Miller, M. E. 1271

Milliez, J. 1261

Mills, J. L. 1504

Milner, A. D. 22

Minerals 847

Miranda, A. F. 1334

Mitogens 38

Mitzner, W. 235

Momma, K. 19

Monnens, L. A. H. 284

Monocyte 1287

5'-Monodeiodinase 1123

Moore, C. F. 1032

Moreau, E. 138

Morishima H. O. 1261

Morphology 262, 269

Morquio disease type A 976

Morrill, C. G. 1520

Morriss, F. H., Jr. 357

Mosaicism 1443

Moser, H. 1411

Moses, S. W. 299

Mossay, C. 1433

Moxey, P. C. 245

M. pneumoniae 813

Mucociliary transport 120

Mucolipidosis I, II, and III 70, 1334

Mucopolysaccharidose IV A 976

Mucosal barrier 241, 245

Mucotaneous candidiasis 1287 
Muir, H. 959

Mukherjee, A. B. 1063

Muller, D. P. R. 1073

Murray, B. N. 916

Muscle 299, 1105,1334

Muscle fiber 128

Mutation 952

Myocardium 349, 849, 967, 1083, 1363

Myosin 229

Mytilus edulis 219

Nadler, H. L. 839

Naftolin, F. 169

Natural cord rupture 53

Natural feeding 245

Neal, P. 1492

Necrotizing enterocolitis 1229

Neely, K. 66

Nelson, T. E. 940

Neonatal cardiac output 1500

Neonatal oxygen consumption 1500

Neonate 10, 66, 262, 295, 794, 859, 912, 1015, 1271,1495

Neonates, mechanically-ventilated 1468

Nephrotic syndrome 340

Nesidioblastosis 1345

Neufeld, N. D. 1058

Neuroblastoma 1117, 1282

Neurofibromatosis 290

Neutral lipids 313

Neutrophil 47, 794, 813, 1271, 1314

Newborn 1429

Niall, M. 1370

Nielsen, H. C. 1245

Niermeijer, M. F. 284

Nimmo, I. A. 1425

Nishihara, S. 19

Nitrogen 1040

Nitrogen metabolism 1454

Nogami, H. 330

Non-age-dependent enzymes 133

Nooijen, J. L. 284

Norepinephrine 99, 912

5 '-Nucleotidase 1411

Nützenadel, W. 309

Nyhan, W. L. 1447

Oakes, G. 163

Obesity 1381

Odell, G. B. 1009

Oesophageal pressures 22

Oh, W. 1348

Olegard, R. 899

Oligodendrocyte 319

Olsen, W. A. 1009

Olsson, T. 964

Omara, P. 1429

Ontogeny 138, 152, 282, 290, 304

Oohira, A. 330

Oparil, S. 1088

Open heart surgery 1024

Opsonin 1256

Opsonization 1525

O'Regan 926

Organs 284

Orloff, S. 1063

Orotic aciduria 115

Os ilium 330

Oshima, R. G. 1492

Oto, Y. 19

Otterbreit, M. J. 95
Overturf, G. D. 107

Owen, G. M. 822

Oxygen $843,967,999$

Oxygen consumption 1111

Oxygen, singlet 956

Oxygen tension 809

Oxygen tension, fetal 1261

Oxygen toxicity 1293

Ozere, R. 1287

Padbury, J. F. 1483

Palmer, A. E. 235

Palmer, S. 163

Palmieri, G. M. A. 1478

Pancreas 1240, 1308

Pancreatic enzymes 250

Pancreatic insufficiency 1051,1351

Pancreozymin 1240

Pancytopenia 95

Pang, K. 241, 245

Pantetheinase 1063

Papadopoulou, Z. L. 887

Papageorgion, G. C. 1019

Papazafiratou, C. 1406

Parathyroid hormone 803, 887, 1134, 1359

Parenteral feeding 1040

Parini, R. 304

Parisi, V. 1429

Parker, P. H. 985

Parks, P. A. 1274

Parotid gland 1439

Parr, G. V. S. 1024

Paiten, J. 362

Partin, J. 362

Parvy, P. 974

Pash, M. 44

Pathak, R. M. 112

Paxson, C. L., Jr. 905

Pediatritian 1319

Pegorier, J-P. 1340

Pencharz, P. 1040

Penn, A. S. 1334

Peptidase 991

Peptide absorption 309

Perelman, A. 1483

Perinatal nutrition 245

Peripheral nerve 295

Peritoneal dialysis 853

Perkins, J. P. 1328

Perman, J. A. 1229

Persistent fetal circulation syndrome 147

Peters, K. 1415

Peterson, L. J. 916

Phagocytic cells 813

Phelps, D. L. 1256

Phenobarbital 1488

Phenylalanine 309

Phenyl-n-methyl transferase 912

Phophaturia 1359

Phosphatidylcholine 866, 1274

Phosphoenolpyruvate carboxykinase 138

Phosphofructokinase 34

Phospholipids 112, 787, 1029

Phosphorus 803, 1100

Phosphorylase kinase deficiency 299

Phototherapy 345, 952, 956, 1019, 1517

Pitts, S. M. 1293

Piu, P. 1443

Placenta 357, 798

Placental blood flow 152

Plasma 14
Plasma proteins 1425

Plasma renin activity 163

Plasma volume 1024

Platzker, C. G. 1422

PMN myosin light chain 229

Pneumonia 908

$\mathrm{PO}_{2} 143$

Polin, R. A. 952, 1495

Polinsky, M. S. 853

Polma, S. H. 14

Polymorphonuclear leukocytes 229

Polymorphonuclear neutrophils 1287

Polysaccharide 892

Pomerance, H. H. 1390

Portacaval shunt 58

Positive pressure ventilation 829

Potashnik, R. 299

Potassium 163, 916, 1134

Potau, N. 798

Powell, S. H. 1234

Pratt, H. 295

Prebis, J. W. 853

Prenatal diagnosis 254

Prieur, A-M. 1266

Probenecid 1447

Prosmanne, J. 1500

Prostaglandin 147, 278, 1293

Prostaglandin $E_{1} 1328,1439$

Prostaglandin $F_{2} 53$

Prostaglandin $I_{2} 971$

Prostaglandin synthetase inhibitors 926

Protein 335, 847, 916, 1040

Protein-calorie malnutrition 930

Protein, plasma 1425

Proteinuria 340

Prothrombin 1045

Pseudomonas aeruginosa 14

Puberty 157, 169, 1488

Pulmonary function 1520

Pulmonary mechanics 979

Putet, G. 803, 1077

Pyrazinamide 1447

Quaife, M. A. 905

Quiet sleep 879, 1055

Quinton, P. M. 366

Rabier, D. 974

Radiant warmer power 1495

Raghunathan, R. 1271

Rajantie, J. 115

Ramanathan, S. 1359

Ravindranath, Y. 95

Red blood cell volume 905

Reed, M. D. 1234

Reference boy 847

Reichman, B. L. 1077

REM 879, 1055

Renal blood flow 1248

Renal clearance values 304

Renal failure 1492

Renin 1088, 1419

Research 1319

Research, pediatric 1465

Reserpine 1308

Residual $\beta$-cell function 62

Respiration 879

Respiratory distress syndrome 1058

Respiratory failure 1495

Respiratory jacket 22

Respiratory pattern 1509 
Response time 143

Retinopathy 1293

Retrolental fibroplasia 1293

Reye's syndrome 362, 1097

Rezunia, I. 6

Riboflavin 956

Rich, K. C. 38

Richard, M. O. 787

Riegel, K. P. 53

Riemenschneider, T. A. 349

Rigatto, H. 1509

Ritchie, D. G. 1492

Riudor, E. 798

Roberts, J. M. 1083

Roberts, R. J. 999

Robertson, B. 833

Robillard, J. E. 1045, 1248

Robins, R. A. 38

Robson, A. M. 916

Rojas, J. 892, 899

Rolin, W. M. 859

Roma, E. 1073

Rooney, S. A. 1274

Rosenfeld, C. R. 874

Rosenthal, A. 1397

Rosso, P. 152

Roth, J. 1345

Rothberg, A. D. 1468

Rubenstein, D. 829

Rudolph, A. M. 843

Sabato, A. R. 813

Saintonge, J. 152

Salbutamol 1433

Salle, B. L. 803

Sandberg, K. 964

Sanderson, M. J. 219

Sandler, P. 887

Sandru, L. 319

Sanfilippo A syndrome 959

Sankaran, K. 1415

Sanna, G. 1443

Sarnaik, A. 95

Sangstad, O. D. 1140

Sbrana, G. 1517

Scarpelli, E. M. 870

Schaffer, T. 319

Schneider, J. A. 1492

Schuit, K. E. 47

Schulman, J. D. 1063

Schulze, K. 1111

Schwarz, H. P. 319

Schwarz, K. B. 1473

Sciacca, R. 1111

Scott, R. 235

Secretin 1240

Segall-Blank, M. 1123

Seger, R. A. 1533

Sell, S. 892

Sepsis 908

Serum immunoglobins 219

Serum levels 50

Sessions, C. 1248

Sex 123, 1032

Sex difference 1245

Shaffer, T. H. 829

Shannon, D. C. 1402

Shanske, S. 1334

Shaw, J. C. L. 1454

Sheep 1248

Shrager, H. 1248
Shwackman's syndrome 1073, 1351

Sialic acid 839

Sideris, E. G. 1019

Siegel, S. R. 163, 1419

Siegrist, H. P. 319, 1411

Silva, O. L. 1032

Sjoqvist, B-A. 964

Skovberg, H. 214

Sleep 367

Sleep, active 1111

Sleep, quiet 1111

Sleigh, M. A. 219

Sloan, H. R. 250

Sly, W. S. 70

Smith, G. J. W. 1274

Smith, J. M. 1077

Snider, R. H. 1032

Snyder, E. L. 794

Sodium 163, 340, 916, 1100, 1134, 1439

Sodium transport 926

Sodoyez, J. C. 1303

Sodoyez-Goffaux, F. R. 1303

Somatosensory potential 295

Sorensen, R. U. 14

Soy formula 1240

Sparks, J. W. 1340

Specificity 940

Speck, W. T. 952, 956

Sphengomyelin 996

Spina bifida 326

Sputum viscoelasticity 120

Stahlman, M. T. 892, 899

Stambouly, J.

Standaert, T. A. 367

Stanley, C. A. 1504

Stapleton, F. B. 1447, 1513

Staphylococcus aureus 952

Starfield, B. 1

Stark, R. I. 1261

Starr, A. 295

Starvation 128, 313, 859

Steele, R. W. 908

Steen-Johnsen, J. 1134

Stefanski, M. 1111

Stein, B. 335

Stellato, A. 991

Stem cell 95

Steranka, B. H. 1100

Sterchi, E. E. 884

Stern, R. C. 14, 1234

St. Geme, J. W. 201

Stiehm, E. R. 38, 1256

Stokes, G. M. 22

Stomach 921

Strange, R. C. 1425

Strauss, R. G. 794, 1314

Stress, thermal 1111

Stress, surgical 1340

Strife, C. F. 362

Strömberg, P. 254

Strømme, J. H. 1134

Strunk, R. 107

Stuart, M. J. 971

Subrahmanyam, D. 112

Sucrase 1068

Sucrase-isomaltase deficiency 214

Sudar, K. 50

Sudden infant death syndrome, 879, 1055, 1245,1402

Suh, S. M. 1359

Sulfamethoxazole 1533
Sulfatide synthesis 319

Sundell, H. 892

Superoxidase dismutase 1356

Surface pressure 1029

Surfactant 235, 866, 996, 1029, 1245

deficiency 833

Surface active material flux 1422

Surgery, open heart 1024

Surgical stress 1340

Susa, J. B. 1348

Suzuki, F. 330

Sueger, T. 254

Swenningsen, N. W. 979

Swyer, P. R. 1077

Sympatho-adrenal system 1483

Taurine 10

Technetium-99m 905

T lymphocytes 1266

Terbutaline 1433

Tetracycline 817

Thack, B. T. 143

Theophylline 304, 870

Therapy, steroid 1520

Therapy, theophylline 1520

Thong, Y. H. 813

Thorax 833

Thrombosis 1015

Thulin, G. E. 262

Thurlbeck, W. M. 269

Thyroxine 6, 1123

Tidal volume 22

Tina, L. U. 887

Tissue weight 128

Torday, J. S. 1245

Tordet, C. 787

Towne, D. 1063

Toxin 892

Trace elements 284

Trachea 269, 817

Tracheal fluid 1422

Transient erythroblastopenia of childhood 1036

Trauner, D. A. 1097

Travis, S. F. 34, 133

Trentalange, M. J. 123

Trier, J. S. 245

Triiodothyronine 6

3,5,3'-Triiodothyronine 1123

Trimethoprin 1533

Tripp, J. H. 1073

Trypsin 1351

Tsang, R. C. 66

Tsuda, K. 330

Tuchschmid, P. E. 28,1400

Tweed, W. A. 44

Type I glycogen storage disease 1504

Type III group B streptococci 1525

Udall, J. N. 241, 245

Umbilical cord 1483

Undernutrition 112, 169

Urea cycle 115

Uremia 853

Uric acid 1447, 1513

Urine calcitonin 1032

Valk, T. W. 157

Van Ameringen, M. R. 967, 1500

Vanbell, E. 1248

Van de Wiele, R. 1261 
Van Den Berghe, G. 58

Van den Hamer, C. J. A. 284

Vannier, J. P. 326

Van Thiel, D. H. 335

Vascular permeability 899

Vasopressin 1261

Vecchi, C. 1517

Venous pressure 874

Ventilation 367, 1111

Ventilatory chemoreceptor response 1402

Ventilatory drive 1520

Ventricle 349

Verellen, G. J. E. 1077

Vermeulen, M. W. 1234

Vertebral development 326

Vidyasagar, D. 1406

Visual evoked potential (VEP) 1429

Vitamin D 1134

Vitamin E 262, 971

Vitsa, E. M. 1019

Volume dilution 905

Voorhess, M. L. 99, 912
Wachlicht-Rodbard, H. 1345

Wada, N. 1266

Wajer, S. D. 1293

Walker, W. A. 241, 245

Walker Smith, G. J. 262

Wallace, K. B. 1088

Walser, K. 53

Walsh, E. M. 1055

Warburton, D. 1348, 1422

Warshaw, D. B. 262

Waters, L. A. 1229

Wavelength 1019

Weber, P. C. 278

Weight 123, 1390

Weight reduction 1381

Weismann, D. N. 1248

Wender, D. F. 262

Wheeler, J. M. 874

Whelan, R. D. H. 1117

Whitington, P. F. 345, 1009

Whitsett, J. A. 1363

Wiesmann, U. 319, 1411
Wilkinson, A. W. 313

Wilen, R. 169

Willemse, J. 284

Williams D. F. 1093

Wilson, S. R. 1525

Wolfish, N. 282

Woodman, D. 1128

Woodrum, D. E. 367

Woods, J. R., Jr. 1429

Work of breathing 22

Wu, J. T. 50

Yaffe, S. J. 1488

Yang, H. H. 229

Yee, E. S. 1229

Young, M. K. 1105

Zelkowitz, M. 290

Ziegler, E. E. 847

Zinc 985

Zuppenger, K. 319

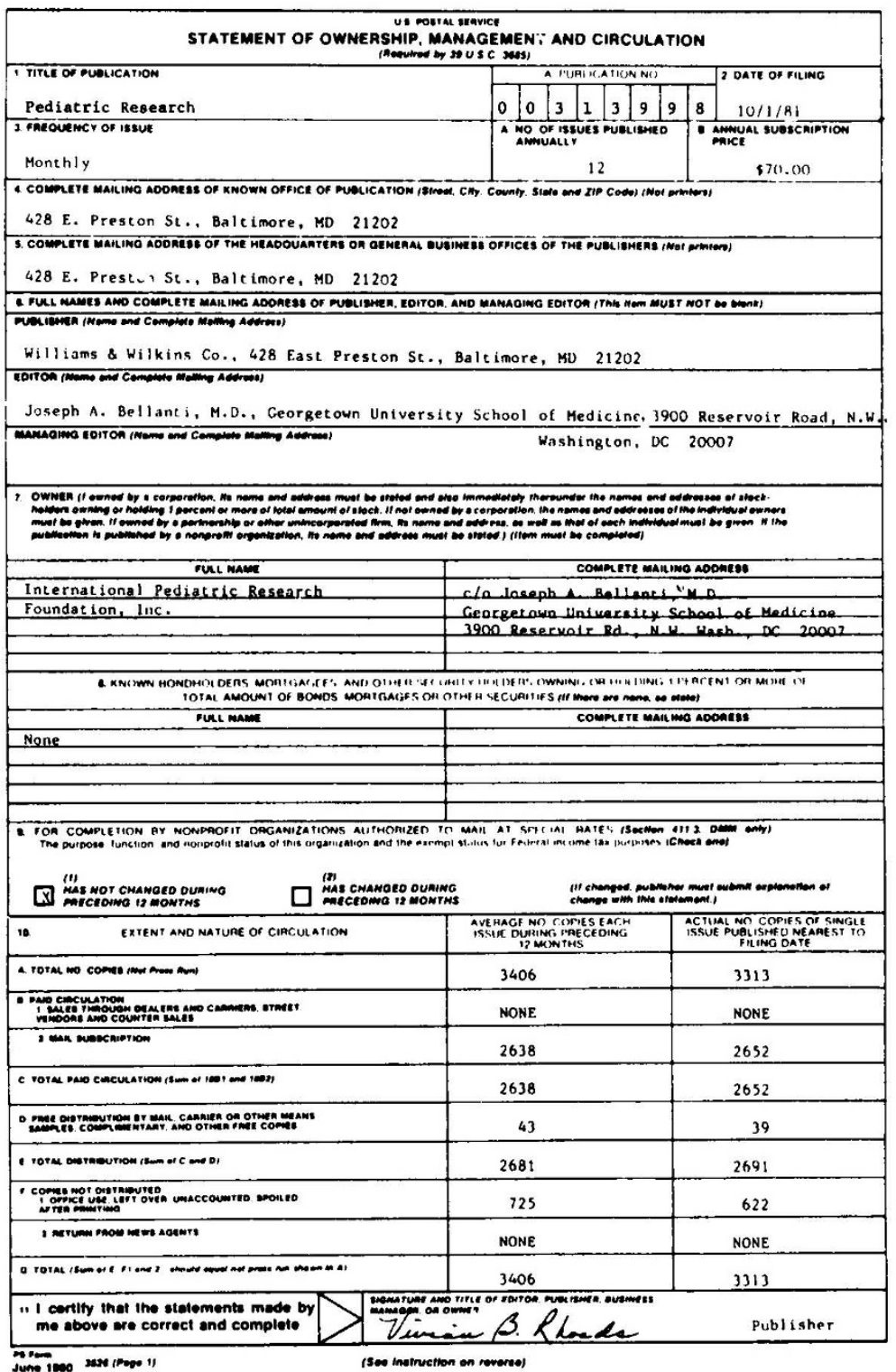

